\title{
GENERIC DYNAMICS AND MONOTONE COMPLETE $C^{*}$-ALGEBRAS
}

\author{
DENNIS SULLIVAN, B. WEISS AND J. D. MAITLAND WRIGHT
}

\begin{abstract}
Let $R$ be any ergodic, countable generic equivalence relation on a perfect Polish space $X$. It follows from the main theorem of $\S 1$ that, modulo a meagre subset of $X, R$ may be identified with the relation of orbit equivalence ensuing from a canonical action of $\mathbf{Z}$.

Answering a longstanding problem of Kaplansky, Takenouchi and Dyer independently gave cross-product constructions of Type III $A W^{*}$-factors which were not von Neumann algebras. As a specialization of a much more general result, obtained in $\S 3$, we show that the Dyer factor is isomorphic to the Takenouchi factor.
\end{abstract}

Introduction. Our two main results are Theorems 1.8 and 3.4. The first result is concerned with a countable group $G$ acting as homeomorphisms on a complete metric space. When there is a dense $G$-orbit, we show that the relation of orbit equivalence (with respect to $G$ ) can be identified, modulo meagre sets, with that arising from a canonical action of $\mathbf{Z}$.

The other main result, is on the classification problem for $A W^{*}$-cross-products. It follows as a corollary to Theorem 3.4 that the Takenouchi factor is isomorphic to the Dyer factor.

The intimate relationship between classical dynamics and von Neumann algebras is paralleled by an equally close connection between generic dynamics and monotone complete $A W^{*}$-algebras. However, the reader whose main interest is in the results on countable groups acting on complete separable metric spaces, may safely ignore all references to $C^{*}$-algebras and all references to spaces which are not metric spaces. He could proceed straight to $\S 1$, referring to $\S 0$ for any unfamiliar notation or results.

Let $G$ be a countable discrete group acting as homeomorphisms of a perfect Polish space $X$ (that is, $X$ is homeomorphic to a complete separable metric space without isolated points). This action is said to be generically ergodic if, for some $x_{0} \in X$, the orbit $G x_{0}$ is dense in $X$. Equivalently, each $G$-invariant Borel subset of $X$ is either meagre or the complement of a meagre set.

From the standpoint of topological dynamics, the action of $G$ on $X$ would be studied without throwing anything away. In classical dynamics, sets which are null with respect to some $G$-invariant (or quasi-invariant) measure are regarded as negligible. In generic dynamics we investigate the action of $G$ on $X$ modulo meagre sets. We prove, in Theorem 1.8 , that if $G$ acts (generically) ergodically then orbit

Received by the editors April 23, 1985 and, in revised form, June 18, 1985.

1980 Mathematics Subject Classification. Primary 46L99, 28D99, 54H20.

Key words and phrases. Dynamics, orbit equivalence, countable group, monotone crossproducts, groupoid $A W^{*}$-algebras, Takenouchi factor, Dyer factor. 
equivalence on $X$ is unique, modulo meagre sets. This is astonishingly different from what happens in classical dynamics.

For, in classical dynamics, even when $G=\mathbf{Z}$, different actions of $\mathbf{Z}$ give rise to a continuum of, essentially different, orbit equivalence relations. (They can be classified into Types $\mathrm{II}_{1}, \mathrm{II}_{\infty}$ and $\mathrm{III}_{\lambda}$, with $0 \leq \lambda \leq 1$ ) On the other hand, any action of an amenable group [1] is orbit equivalent to an action of $\mathbf{Z}$. But, in general, nonamenable groups give rise to orbit equivalence relations which do not arise from actions of $\mathbf{Z}$.

Let $B$ be the algebra of bounded Borel functions on the unit interval and $M$ the ideal of Borel functions $f$ for which $\{x: f(x) \neq 0\}$ is meagre. Then $B / M$ is a commutative $A W^{*}$-algebra which is not a von Neumann algebra.

When $G$ is a countable group acting freely and ergodically on $B / M$, there is a corresponding monotone complete $C^{*}$-algebra, the monotone cross-product $M(B / M, G)$. This is an $A W^{*}$-factor of Type III which is not a von Neumann algebra [11].

The existence of $A W^{*}$-factors which are not von Neumann algebras was a question of Kaplansky which went unanswered for many years. Independently, Takenouchi and Dyer gave examples, see $[2, \mathbf{1 1}, \mathbf{1 5}]$, which were of the form $M\left(B / M, G_{1}\right)$ and $M\left(B / M, G_{2}\right)$ for different abelian groups $G_{1}$ and $G_{2}$. By Corollary 3.5 the Takenouchi and Dyer algebras are isomorphic. But much more is true. In startling contrast to the situation for von Neumann algebras, we show in Theorem 3.4 that all $A W^{*}$-factors of the form $M(B / M, G)$ are isomorphic.

Inspired by Feldman and Moore's $[\mathbf{4}, \mathbf{5}]$ construction of groupoid von Neumann algebras associated with measurable countable equivalence relations, we consider an analogous construction of a groupoid $A W^{*}$-algebra associated with an equivalence relation modulo meagre sets in $\S 2$. It would be possible to go into considerable detail, but we confine ourselves to the fragment of this theory which we need in $\S 3$.

0. Preliminaries and background information. Let $X$ be a Polish space, that is, a topological space which is homeomorphic to a complete separable metric space. Let $\operatorname{Bor}(X)$ be the $\sigma$-field of Borel subsets of $X$, and let $\operatorname{Mg}(X)$ be the ideal of all meagre Borel subsets of $X$. Then, by a theorem of Birkhoff and Ulam $[\mathbf{1 3}, \mathrm{p}$. 75], $\operatorname{Bor}(X) / \mathrm{Mg}(X)$ is isomorphic to the complete Boolean algebra of regular open subsets of $X$.

Let $\left(U_{n}\right)(n=1,2, \ldots)$ be a sequence of nonempty open sets which form a base for the topology of $X$. For each $n$, let $V_{n}$ be the interior of the closure of $U_{n}$. Clearly each regular open subset of $X$ contains $V_{n}$, for some $n$.

We shall assume in all that follows, that the Polish space $X$ is perfect, that is, has no isolated points. This is equivalent to the Boolean algebra $\operatorname{Bor}(X) / \mathrm{Mg}(X)$ being nonatomic. It turns out that $\operatorname{Bor}(X) / \operatorname{Mg}(X)$ is isomorphic to $\operatorname{Bor}(\mathbf{R}) / \operatorname{Mg}(\mathbf{R})$; see $[13$, p. 155].

Let $S$ be the Stone structure space of $\operatorname{Bor}(X) / \operatorname{Mg}(X)$. Then $S$ is compact and extremally disconnected. By the remark above, there exists a sequence of nonempty clopen subsets of $S,\left(K_{n}\right)(n=1,2, \ldots)$, such that, given any nonempty clopen set $K \subset S$, there exists an $n$ such that $K_{n} \subset K$. This clearly implies that $S$ has a countable dense set, but, of course, $S$ does not have a countable base.

Let $\theta$ be a bijection of $X$ onto $X$. We shall call $\theta$ a pseudo-homeomorphism of $X$ if $\theta$ is a Borel bijection of $X$ onto $X$ such that $\theta[E]$ is meagre if, and only if, $E$ 
is meagre. Clearly all homeomorphisms are pseudo-homeomorphisms, but simple examples show that Borel bijections need not be pseudo-homeomorphisms.

When $\theta$ is a pseudo-homeomorphism of $X$, then there exists a dense $G_{\delta}$-subset $X_{0}$ such that the restriction of $\theta$ to $X_{0}$ is a homeomorphism of $X_{0}$ onto $X_{0}$. This follows by applying a theorem of Kuratowski $\left[9\right.$, p. 400] to $\theta$ and $\theta^{-1}$.

Let $R$ be an equivalence relation on a Polish space $Y$; we identify $R$ with its graph. When $R$ is a Borel subset of $Y \times Y$ and each equivalence class is countable, then $R$ is said to be a countable standard equivalence relation. For any $A$ contained in $Y$ the saturation of $A$ (by $R$ ) is the set

$$
R[A]=\{y \in Y \text {; there exists } x \in A \text { such that } x R y\} .
$$

When $R$ is a countable standard equivalence relation such that, for each meagre set $M \subset Y$, its saturation $R[M]$ is also meagre, we shall call $R$ a countable generic equivalence relation.

Proposition 0.1. Let $R$ be a countable generic equivalence relation on a Polish space $Y$. Then there exists a countable group, $\Gamma$, of pseudo-homeomorphisms of $Y$ such that

$$
R=\{(x, \gamma x): x \in Y \text { and } \gamma \in \Gamma\} .
$$

Furthermore, there exists a dense $G_{\delta}$-set $Y_{0} \subset Y$ and a countable group, $G$, of homeomorphisms of $Y_{0}$ such that $R\left[Y_{0}\right]=Y_{0}$ and

$$
R \cap\left(Y_{0} \times Y_{0}\right)=\left\{(x, \gamma x): x \in Y_{0} \text { and } \gamma \in G\right\} \text {. }
$$

ProOF. Since $R$ is a countable standard equivalence relation, there exists, by Theorem 1 of [3], a countable group, $\Gamma$, of Borel bijections of $Y$ such that $R=$ $\{(x, \gamma x): x \in Y$ and $\gamma \in \Gamma\}$.

Let $M$ be any meagre subset of $Y$. Then $R[M]$ is meagre. So, for each $\gamma \in \Gamma$, $\gamma[M]$ and $\gamma^{-1}[M]$ are meagre. So $\Gamma$ is a group of pseudo-homeomorphisms. It follows from the properties of pseudo-homeomorphisms and the countability of $\Gamma$, that there exists a $\Gamma$-invariant, dense, $G_{\delta}$-subset $Y_{0} \subset Y$ such that each $Y$ in $\Gamma$ restricts to a homeomorphism of $Y_{0}$ onto itself. Let $G$ be the countable group of such restrictions.

It follows from the above proposition that the study of countable generic equivalence relations reduces to the study of countable groups of homeomorphisms. In the next section we shall investigate the latter but, of course, the main result, Theorem 1.8 , applies to ergodic generic equivalence relations.

For any topological space $T$, let $B(T)$ be the $C^{*}$-algebra of all bounded (complexvalued) Borel functions on $T, M(T)$ the ideal of all $f$ in $B(T)$ for which $\{t \in$ $T: f(t) \neq 0\}$ is meagre and let $C(T)$ be the algebra of all bounded continuous functions on $T$. Then, whenever $X$ is a Polish space with no isolated points,

$$
B(X) / M(X) \approx C(S) .
$$

Following [16] we shall call $C(S)$ the Dixmier algebra and will sometimes denote it by $D$. We remark that $B(S) / M(S)$ is, again, $C(S)$. The selfadjoint part of $C(S)$ is a complete vector lattice. By the Baire Category Theorem the natural map $j$ from $C(X)$ into $B(X) / M(X) \approx C(S)$ is an injection. It is elementary, but useful, to observe that $j[C(X)]$ is order-dense in $C(S)$; that is, each selfadjoint $b$ in $C(S)$ 
is the supremum of $\{j(a): j(a) \leq b$ and $a \in C(X)\}$. In particular, this implies that if two *-automorphisms of $C(S)$ coincide on $j[C(X)]$, then they are identical.

Let $\theta$ be a pseudo-homeomorphism of $X$. Then $f \rightarrow f \circ \theta$ is a $*$-automorphism of $B(X)$ which maps $M(X)$ onto $M(X)$. This induces a *-automorphism of

$$
B(X) / M(X) \approx C(S)
$$

which, in turn, induces a homeomorphism $\bar{\theta}$ of $S$.

Conversely, by a theorem of Maharam and Stone [10] (see [14] for a noncommutative generalization) every *-automorphism of $C(S)$ arises in this way from a pseudo-homeomorphism of $X$.

1. Discrete group actions. Throughout this section $X$ is either a perfect Polish space or $S$, the Stone structure space of $\operatorname{Bor}(\mathbf{R}) / \operatorname{Mg}(\mathbf{R})$, or a dense $G_{\delta^{-}}$ subset of $S$.

LEMMA 1.1. Let $G$ be a countable group of homeomorphisms of $X$. Then the following conditions are equivalent:

(1) Each $G$-invariant Borel subset of $X$ is meagre or comeagre.

(2) Each $G$-invariant open subset of $X$ is either empty or dense.

(3) There exists a $G$-invariant, dense $G_{\delta}$-subset of $X, Y$, such that $\{g y: g \in G\}$ is dense for each $y \in Y$.

(4) There exists an $x_{0}$ in $X$ such that $G x_{0}$ is a dense orbit.

ProOF. It is clear that (1) implies (2).

There exists a sequence of nonempty open sets $\left(U_{n}\right)(n=1,2, \ldots)$ such that each nonempty open set in $X$ contains some $U_{n}$.

We now assume (2) and deduce (3). For each $n$, the set $\bigcup_{g \in G} g\left[U_{n}\right]$ is $G$-invariant, nonempty, and open. So it is dense in $X$.

Let $Y$ be the dense, $G_{\delta}$-set $\bigcap_{n=1}^{\infty} \bigcup_{g \in G} g\left[U_{n}\right]$. Let $y \in Y$. Let $V$ be any nonempty open subset of $X$. For some $n, U_{n} \subset V$. For some $g \in G, g y \in U_{n}$. Hence, $G y$ is a dense orbit in $X$; that is, (2) implies (3).

It is clear that (3) implies (4). We shall now show that (4) implies (2). Let $U$ be any nonempty, $G$-invariant open subset of $X$. Then, by (4), $g_{0} x_{0} \in U$ for some $g_{0} \in G$. Since $U$ is $G$-invariant, it follows that $G x_{0} \subset U$, and so $U$ is dense in $X$.

It remains to show that (2) implies (1).

Let $E$ be a nonmeagre $G$-invariant Borel subset of $X$. By the Baire Property, there exists a nonempty open set $U$ and a meagre set $M_{0}$ such that

$$
E=\left(U \backslash M_{0}\right) \cup\left(M_{0} \backslash U\right) .
$$

Now $M_{0}$ is contained in a $G$-invariant, meagre $F_{\sigma}$-set $M_{1}$. Since (2) implies (3), there exists a $G$-invariant, dense $G_{\delta}$-set, $Y$, such that $Y \subset X \backslash M_{1}$ and each $G$-orbit in $Y$ is dense in $X$.

We observe that $E \cap Y=U \cap Y$. Then, in the relative topology of $Y, U \cap Y$ is a $G$-invariant open set. Since each $G$-orbit in $Y$ is dense in $Y$, it follows from the equivalence of (2) and (3) that $U \cap Y$ is dense in $Y$. Hence $U$ is dense in $X$. So $E$ is the complement of a meagre subset of $X$.

DEFINITION. The action of a countable group, $G$, of homeomorphisms of $X$ is said to be (generically) ergodic if each $G$-invariant open subset of $X$ is either empty or meagre. 
COROLlaRY 1.2. Let $G$ and $X$ be as above. Let $Z$ be a $G$-invariant, dense, $G_{\delta}$-subset of $X$. Then the action of $G$ on $X$ is ergodic if, and only if, the action of $G$ on $Z$ is ergodic.

Proof. Suppose $G$ acts ergodically on $X$. Then there is a dense $G_{\delta}$-subset $Y$, where $Y$ is $G$-invariant, such that $G y$ is dense in $X$ for each $y \in Y$. Then $Y \cap Z$ is a dense $G_{\delta}$-subset of $Z$ such that, for each $y \in Y \cap Z, G y$ is a dense orbit in $Z$. So $G$ acts ergodically on $Z$. The converse is clear.

COROLLARY 1.3. Let $G$ be a countable group of pseudo-homeomorphisms of $X$. Suppose that each $G$-invariant Borel subset of $X$ is either meagre or comeagre. Let $Y$ be a $G$-invariant, dense $G_{\delta}$-subset of $X$ such that each $g$ in $G$ restricts to a homeomorphism of $Y$ onto $Y$. Then the action of $G$ on $Y$ is ergodic.

When $G$ is a countable group of pseudo-homeomorphisms of $X$, such that every $G$-invariant Borel set is either meagre or comeagre, the action of $G$ is said to be (generically) ergodic.

LEMMA 1.4. Let $G$ be a countable group of homeomorphisms of $X$. Then there exists a $G$-invariant, dense, $G_{\delta}$-subset $Y$ such that, for each $g \in G$, the set

$$
F(g)=\{y \in Y: g(y)=y\}
$$

is clopen in the relative topology of $Y$.

ProOF. Let $F^{*}(g)$ be the closed set $\{x \in X: g(x)=x\}$. Let $F^{\circ}(g)$ be the interior of $F^{*}(g)$. Let $M_{1}$ be the meagre $F_{\sigma}$-set $\bigcup_{g \in G}\left(F^{*}(g) \backslash F(g)\right)$. Let $M_{2}$ be the saturation of $M_{1}$ by $G$; that is,

$$
M_{2}=\bigcup_{g \in G} g\left[M_{1}\right]
$$

Let $Y=X \backslash M_{2}$. Then, for each $g$,

$$
F(g)=F^{*}(g) \cap Y=F^{\circ}(g) \cap Y .
$$

So $F(g)$ is both closed and open in the relative topology of $Y$.

The following notion is important for some applications to $C^{*}$-algebras. Let $G$ be a group of homeomorphism acting on $X$. Let $h$ be a pseudo-homeomorphisms of $X$ onto itself. Then $h$ is said to be $G$-decomposable over $X$ if there exists a sequence of pairwise disjoint clopen sets $\left(K_{j}\right)(j=1,2, \ldots)$ and a sequence $\left(g_{n}\right)(n=1,2, \ldots)$ in $G$ such that $\bigcup K_{j}$ is dense in $X$ and, for each $x \in K_{j}, h(x)=g_{j}(x)$. If $\bigcup K_{j}$ is the whole of $X$, then we say that $h$ is strongly $G$-decomposable over $X$. When this occurs, $h$ must be a homeomorphism.

Let $G$ and $\Gamma$ be countable groups of homeomorphisms of $X$. If each $\gamma \in \Gamma$ is strongly $G$-decomposable over $X$ and each $g \in G$ is strongly $\Gamma$-decomposable over $X$, we say that the $G$ and $\Gamma$ actions are strongly equivalent. Clearly when $G$ and $\Gamma$ are strongly equivalent, Graph $G=\operatorname{Graph} \Gamma$; that is, $G$ and $\Gamma$ are orbit equivalent.

It is convenient to introduce a weaker notion of equivalence which is applicable when $G$ and $\Gamma$ act on different spaces. Let $G$ be a countable group of pseudo-homeomorphisms acting on $X_{1}$, and let $\Gamma$ be a countable group of pseudohomeomorphisms acting on $X_{2}$. We shall say that the $G$-action on $X_{1}$ is equivalent to the $\Gamma$-action on $X_{2}$ when the following conditions are satisfied. There exists a 
dense $G_{\delta}$-subset $Y_{j} \subset X_{j}(j=1,2)$, and there is a homeomorphism $\pi$ from $Y_{1}$ onto $Y_{2}$. For each $g \in G$, the restriction of $g$ to $Y_{1}$ is a homeomorphism of $Y_{1}$ onto itself. For each $\gamma \in \Gamma$, the restriction of $\gamma$ to $Y_{2}$ is a homeomorphism of $Y_{2}$ onto itself. Further, the action of $G$ on $Y_{2}$ given by $\left\{\pi g \pi^{-1}: g \in G\right\}$ is strongly equivalent to the action of $\Gamma$ on $Y_{2}$.

LEMMA 1.5. Let $G$ and $\Gamma$ be countable groups of homeomorphisms of $X$. Let each $\gamma \in \Gamma$ be strongly $G$-decomposable over $X$. For each $g \in G$, let $\{x \in X: g(x)=$ $x\}$ be a clopen set.

Let $\Delta$ be a countable dense subset of $X$, which is $G$-invariant and $\Gamma$-invariant, and such that $\Delta \times \Delta \subset$ Graph $G$ and $\Delta \times \Delta \subset$ Graph $\Gamma$.

Then there exists a dense $G_{\delta}$-set $Y$, with $\Delta \subset Y \subset X$, which is $G$-invariant and $\Gamma$-invariant and such that $G$ and $\Gamma$ are strongly equivalent on $Y$.

PROOF. Let $g$ be any element of $G$. Fix $x_{0} \in \Delta$. Then

$$
\left(x_{0}, g x_{0}\right) \in \Delta \times \Delta \subset \operatorname{Graph} \Gamma .
$$

So, for some $\gamma_{1} \in \Gamma, g\left(x_{0}\right)=\gamma_{1}\left(x_{0}\right)$. Since $\gamma_{1}$ is strongly $G$-decomposable over $X$, there exists a clopen neighbourhood of $x_{0}, K_{0}$, and $g_{0} \in G$ such that $g_{0}(x)=\gamma_{1}(x)$ for all $x \in K_{0}$. Since $g^{-1} g_{0}\left(x_{0}\right)=x_{0}$, there is a clopen neighbourhood of $x_{0}, K_{1} \subset$ $K_{0}$, such that $g(x)=g_{0}(x)=\gamma_{1}(x)$ for all $x \in K_{1}$. Since $\Delta$ is countable we can find a sequence of pairwise disjoint clopen sets $\left(K_{n}\right)(n=1,2, \ldots)$ and a sequence $\left(\gamma_{n}\right)(n=1,2, \ldots)$ in $\Gamma$ such that

$$
g(x)=\gamma_{n}(x) \text { for all } x \in K_{n}
$$

and $\Delta \subset \bigcup_{1}^{\infty} K_{n}$.

Let $O_{g}$ be the dense open set $\bigcup_{1}^{\infty} K_{n}$. Let $Y_{0}$ be the dense $G_{\delta}$-set $\bigcap_{g \in G} O_{g}$. Let $Y$ be the intersection of

$$
\left\{\rho\left[Y_{0}\right]: \rho \text { is in the group generated by } G \text { and } \Gamma\right\} .
$$

Then $Y$ is a dense $G_{\delta}$-set containing $\Delta$. Also $G$ and $\Gamma$ are strongly equivalent on $Y$.

The following technical lemma is crucial.

LEMMA 1.6. Let $G$ be a countable group of homeomorphisms acting ergodically on $X$, and let $X$ be totally disconnected. Let $\Delta=\left\{t_{n}: n=0,1,2, \ldots\right\}$ be a dense orbit of $G$. Let $A$ and $B$ be nonempty, disjoint clopen subsets of $X$. Let $a \in A \cap \Delta$ and $b \in B \cap \Delta$. Then there exists a $G$-invariant, dense $G_{\delta}$-set $Y$, with $\Delta \subset Y$, and a homeomorphism $h$ from $Y$ onto $Y$, with the following properties. First $h$ interchanges $A \cap Y$ and $B \cap Y$ and $h$ is constant on $y \backslash(A \cup B)$. Secondly, $h(a)=b$ and $h=h^{-1}$. Thirdly, $h$ is strongly $G$-decomposable over $Y$.

Proof. Since $a$ and $b$ are in the same orbit, $\Delta, g_{1} a=b$, for some $g_{1} \in G$. Let $A_{1}$ be a clopen neighbourhood of $a$, with $A_{1}$ a proper subset of $A$ and $g_{1}\left[A_{1}\right]$ a proper subset of $B$. Let $B_{1}=g_{1}\left[A_{1}\right]$.

Since $X$ has no isolated points, $A \cap \Delta$ and $B \cap \Delta$ are countably infinite. We enumerate these sets. Let $a_{2}$ be the first term in the enumeration of $A \cap \Delta$ which is not in $A_{1}$. Let $b_{2}$ be the first term in the enumeration of $B \cap \Delta$ which is not in $B_{1}$. Then $g_{2} a_{2}=b_{2}$ for some $g_{2} \in G$. Let $A_{2}$ be a clopen neighbourisood of $a_{2}$ such that $A_{2}$ is a proper subset of $A \backslash A_{1}$ and $g_{2}\left[A_{2}\right]$ is a proper subset 
of $B \backslash B_{1}$. Let $B_{2}=g_{2}\left[A_{2}\right]$. Proceeding inductively (or more precisely, using the axiom of dependent choice) we generate pairwise disjoint sequences of clopen sets $\left(A_{j}\right)(j=1,2, \ldots)$ and $\left(B_{j}\right)(j=1,2, \ldots)$, where $\Delta \cap A \subset \bigcup_{1}^{\infty} A_{j} \subset A$ and $\Delta \cap B \subset \bigcup_{1}^{\infty} B_{j} \subset B$, together with a sequence $\left(g_{n}\right)(n=1,2, \ldots)$ in $G$ such that $g_{n}\left[A_{n}\right]=B_{n}$ for each $n$. Let $F$ be the $G$-saturation of $(A \cup B) \backslash \bigcup_{1}^{\infty}\left(A_{n} \cup B_{n}\right)$ and let $Y=X \backslash F$.

For $x \in A_{n} \cap Y$ let $h(x)=g_{n}(x)$.

For $x \in B_{n} \cap Y$ let $h(x)=g_{n}^{-1}(x)$.

For $x \in Y \backslash(A \cup B)$ let $h(x)=x$.

Then $h$ has all the required properties.

The next lemma is cumbersome to state, but the essential idea is straightforward. We are manufacturing a copy of the dyadic group $\bigoplus \mathbf{Z}_{2}$ from the action of $G$ and at the same time splitting $T$ into dyadic pieces.

LEMMA 1.7. Let $T$ be a totally disconnected, perfect Polish space. Let $G$ be a countable group of homeomorphisms acting ergodically on $T$. Let $\Delta=\left\{t_{0}, t_{1}, \ldots\right\}$ be a dense orbit. Let $\left(S_{k}\right)(k=1,2, \ldots)$ be a monotone decreasing sequence of clopen neighbourhoods of $t_{0}$ such that $t_{n} \notin S_{n}$ for any $n$. Then the following statements hold.

(1) There exists a monotone decreasing sequence of $G$-invariant, $G_{\delta}$-sets, $\left(T_{n}\right)(n$ $=1,2, \ldots)$, where $\Delta \subset T_{n}$ for each $n$.

(2) There is a sequence $\left(h_{n}\right)(n=1,2, \ldots)$, where each $h_{n}$ is a homeomorphism of $T_{n}$ onto $T_{n}$ and $h_{n}=h_{n}^{-1}$. For $1 \leq k \leq n$ the functions $h_{k} \mid T_{n}$ are mutually commutative. Each $h_{n}$ is strongly $G$-decomposable over $T_{n}$.

(3) For each positive integer $n$, there exists a family of pairwise disjoint, clopen subsets of $T_{n}$,

$$
\left\{K^{n}\left(\alpha_{1}, \alpha_{2}, \ldots, \alpha_{n}\right):\left(\alpha_{1}, \alpha_{2}, \ldots, \alpha_{n}\right) \in\{0,1\}^{n}\right\}
$$

whose union is $T_{n}$.

(4)

$$
\begin{aligned}
K^{n}\left(\alpha_{1}, \alpha_{2}, \ldots, \alpha_{n}\right) \cap T_{n+1}= & K^{n+1}\left(\alpha_{1}, \alpha_{2}, \ldots, 0\right) \\
& \cup K^{n+1}\left(\alpha_{1}, \alpha_{2}, \ldots, \alpha_{n}, 1\right) .
\end{aligned}
$$

(5) $K^{n}(\underline{0}) \subset S_{n} \cap T_{n}$ and $t_{0} \in K^{n}(\underline{0})$.

(6) Let $\underline{\alpha} \in\{0,1\}^{n}$. Then the homeomorphism $h_{1}^{\alpha_{1}} h_{2}^{\alpha_{2}} \cdots h_{n}^{\alpha_{n}}$ acting on $T_{n}$ interchanges $K^{n}(\underline{0})$ with $K^{n}(\underline{\alpha})$.

(7) For each n,

$$
\left\{t_{0}, t_{1}, \ldots, t_{n}\right\} \subset\left\{h_{1}^{\alpha_{1}} h_{2}^{\alpha_{2}} \cdots h_{n}^{\alpha_{n}}\left(t_{0}\right): \underline{\alpha} \in\{0,1\}^{n}\right\} .
$$

ProOF. We proceed inductively. Lemma 1.6 gives the first step.

Let $A=S_{1}$ and $B=T \backslash S_{1}$. So $t_{0} \in A$ and $t_{1} \in B$. By the preceding lemma, there exists a dense $G_{\delta}$-set $T_{1} \subset T$ such that $\Delta \subset T_{1}$ and a homeomorphism $h_{1}$ from $T_{1}$ onto $T_{1}$ which is strongly $G$-decomposable over $T_{1}$, interchanges $A \cap T_{1}$ and $B \cap T_{1}$ and maps $t_{0}$ to $t_{1}$. Also $h_{1}=h_{1}^{-1}$. Let $K^{1}(0)=A \cap T_{1}$ and $K^{1}(1)=B \cap T_{1}$.

Let us now suppose that we have constructed $\left(T_{1}, \ldots, T_{n}\right)$ and $\left(h_{1}, \ldots, h_{n}\right)$ and the families $\left\{K^{k}(\underline{\alpha}): \underline{\alpha} \in\{0,1\}^{k}\right\}$ for $k=1,2, \ldots, n$. We wish to make the $(n+1)$ th step of this inductive construction.

For some $\underline{\alpha} \in\{0,1\}^{n}, t_{n+1} \in K^{n}\left(\alpha_{1}, \alpha_{2}, \ldots, \alpha_{n}\right)$. Let $c=h_{1}^{\alpha_{1}} h_{2}^{\alpha_{2}} \cdots h_{n}^{\alpha_{n}}\left(t_{n+1}\right)$. Then $c \in K^{n}(\underline{0})$. If $c \neq t_{0}$, let $b=c$. If $c=t_{0}$, let $b$ be any element of $K^{n}(\underline{0}) \cap \Delta$ 
other than $t_{0}$. Let $A$ be a clopen subset of $K^{n}(\underline{0}) \cap S_{n+1}$ such that $t_{0} \in A$ and $b \notin A$. Let $B=K^{n}(0, \ldots, 0) \backslash A$.

We apply Lemma 1.6 to $A \cup B$. Let $Y$ be a $G$-invariant, $G_{\delta}$-subset of $T_{n}$, with $\Delta \subset$ $Y$, and $h$ a homeomorphism of $Y$ onto $Y$, as in Lemma 1.6. In particular, $h\left(t_{0}\right)=b$, $h$ interchanges $A \cap Y$ and $B \cap Y, h=h^{-1}$ and $h$ is strongly $G$-decomposable over $Y$.

Let $T_{n+1}=T_{n} \cap Y$.

Let $K^{n+1}(\underline{0})=A \cap T_{n+1}$ and $K^{n+1}(0,0, \ldots, 0,1)=B \cap T_{n+1}$. For each $\underline{\alpha} \in$ $\{0,1\}^{n}$ we define

$$
K^{n+1}(\underline{\alpha}, 0)=h_{1}^{\alpha_{1}} \cdots h_{n}^{\alpha_{n}}\left[K^{n+1}(\underline{0})\right]
$$

and

$$
K^{n+1}(\underline{\alpha}, 1)=h_{1}^{\alpha_{1}} \cdots h_{n}^{\alpha_{n}}\left[K^{n+1}(0,0, \ldots, 0,1)\right] .
$$

We define $h_{n+1}$ as follows. Let

$$
h_{n+1}(x)=h_{1}^{\alpha_{1}} \cdots h_{n}^{\alpha_{n}} h h_{1}^{\alpha_{1}} \cdots h_{n}^{\alpha_{n}}(x)
$$

for $x \in K^{n}\left(\alpha_{1}, \alpha_{2}, \ldots, \alpha_{n}\right)$. Then it is straightforward to verify that $h_{n+1}$ commutes with $h_{j}$ for $1 \leq j \leq n$ and has all the other required properties. This completes the $(n+1)$ th step of the construction.

We remark that since we may replace each $T_{n}$ by $\bigcap_{n=1}^{\infty} T_{n}$, which is again a dense $G_{\delta}$-set, we may use the preceding lemma with the additional assumption that each $T_{n}=T_{1}$.

We shall identify the Cantor set $C$ with the compact group $\prod \mathbf{Z}_{2}$. The natural action of $\bigoplus \mathbf{Z}_{2}$ on $\Pi \mathbf{Z}_{2}$ is defined by $(h, x) \rightarrow x+h$. Let $\Gamma$ be the group of homeomorphisms of $\prod \mathbf{Z}_{2}$ arising in this way from $\bigoplus \mathbf{Z}_{2}$.

THEOREM 1.8. Let $T$ be a perfect Polish space, and let $G$ be a countable group of homeomorphisms acting ergodically on $T$. Then the $G$-action on $T$ is equivalent to the $\Gamma$-action on the Cantor set.

Proof. Since $T$ has a countable base and $G$ is countable, we can find a $G$ invariant, dense $G_{\delta}$-subset, which is totally disconnected. Since $G_{\delta}$-subsets of Polish spaces are Polish, it follows from Lemmas 1.4 and 1.1 that we may suppose, without loss of generality, that $T$ is totally disconnected, $G t$ is a dense orbit for each $t \in T$ and $\{t \in T: g(t)=t\}$ is a clopen set for each $g \in G$.

Let $\rho$ be a complete metric for $T$.

Fix $t_{0} \in T$ and let $\Delta=G t_{0}$. Let $\left(h_{n}\right)(n=1,2, \ldots)$ and $\left\{K^{n}(\underline{\alpha}): \underline{\alpha} \in\{0,1\}^{n}\right\}$ $(n=1,2, \ldots)$ be constructed as in Lemma 1.7 , where we may suppose that $T=T_{n}$ for all $n$. We also demand that each $S_{n}$ is contained in a sphere of radius $1 / n$ centred on $t_{0}$.

By construction

$$
\left\{t_{0}\right\}=\bigcap_{n=1}^{\infty} K^{n}(\underline{0})
$$

and $\lim _{n \rightarrow \infty} \operatorname{diameter}\left(K^{n}(\underline{0})\right)=0$. For each $\underline{\alpha} \in\{0,1\}^{m}, h_{1}^{\alpha_{1}} h_{2}^{\alpha_{2}} \cdots h_{m}^{\alpha_{m}}$ is a homeomorphism. So

$$
\left\{h_{1}^{\alpha_{1}} \ldots h_{m}^{\alpha_{m}}\left(t_{0}\right)\right\}=\bigcap_{n=m}^{\infty} K^{n}\left(\alpha_{1}, \ldots, \alpha_{m}, 0,0, \ldots, 0\right)
$$


and, if $x_{n} \in K^{n}\left(\alpha_{1}, \ldots, \alpha_{m}, 0,0, \ldots, 0\right)$ for $n \geq m+1$ then

$$
\lim _{n \rightarrow \infty} x_{n}=h_{1}^{\alpha_{1}} h_{2}^{\alpha_{2}} \cdots h_{m}^{\alpha_{m}}\left(t_{0}\right) \text {. }
$$

We now define a map $\pi$ from $T$ into the Cantor set. Let $\left(\alpha_{1}, \alpha_{2}, \ldots\right)$ be any infinite sequence of zeros and ones.

For $x \in \bigcap_{n=1}^{\infty} K^{n}\left(\alpha_{1}, \alpha_{2}, \ldots, \alpha_{n}\right)$ let $\pi(x)$ be $\left(\alpha_{1}, \alpha_{2}, \ldots\right)$. From the definition of the topology of the Cantor set it is clear that $\pi$ is continuous. By Lemma 1.7, and because each $h_{n}$ is strongly $G$-decomposable over $T$, the orbit $\Delta$ coincides with

$$
\left\{h_{1}^{\alpha_{1}} \cdots h_{m}^{\alpha_{m}}\left(t_{0}\right): \underline{\alpha} \in\{0,1\}^{m} ; m=1,2, \ldots\right\} .
$$

It follows from the preceding paragraph that the restriction of $\pi$ to $\Delta$ is a bijection onto $\bigoplus \mathbf{Z}_{2}$, that is, those sequences which take only finitely many nonzero values.

Let $\left(d_{n}\right)(n=1,2, \ldots)$ be a sequence in $\Delta$ and $d \in \Delta$ such that $\lim \pi\left(d_{n}\right)=\pi(d)$. We shall show that $\lim d_{n}=d$.

Let $\pi(d)=\left(\alpha_{1}, \alpha_{2}, \ldots, \alpha_{m}, 0,0, \ldots\right)$. From the definition of the topology of the Cantor set, given any $q$ there exists an $N$ such that, for all $n \geq N, d_{n}$ is in the set

$$
K^{q}\left(\alpha_{1}, \alpha_{2}, \ldots, \alpha_{m}, 0,0, \ldots, 0\right) .
$$

So $h_{1}^{\alpha_{1}} h_{2}^{\alpha_{2}} \cdots h_{m}^{\alpha_{m}}\left(d_{n}\right)$ is in $K^{q}(\underline{0})$ for $n \geq N$. Since $K^{q}(\underline{0})$ is contained in a sphere of radius $1 / q$ centred on $t_{0}$, it follows that $\lim _{n \rightarrow \infty} h_{1}^{\alpha_{1}} h_{2}^{\alpha_{2}} \ldots h_{m}^{\alpha_{m}}\left(d_{n}\right)$ exists and is $t_{0}$. Since $h_{1}^{\alpha_{1}} h_{2}^{\alpha_{2}} \cdots h_{m}^{\alpha_{m}}$ is an idempotent homeomorphism it follows that $\lim _{n \rightarrow \infty} d_{n}$ exists and is $h_{1}^{\alpha_{1}} h_{2}^{\alpha_{2}} \cdots h_{m}^{\alpha_{m}}\left(t_{0}\right)$. That is, $\lim _{n \rightarrow \infty} d_{n}=d$. Thus the restriction of $\pi$ to $\Delta$ is a homeomorphism onto $\bigoplus \mathbf{Z}_{2}$, regarded as a dense subset of $\Pi \mathbf{Z}_{2}$.

By Lavrentiev's Theorem $\left[9\right.$, p. 429, Chapter II] there exists a dense $G_{\delta}$-set $Y$ such that $\Delta \subset Y \subset T$ and $\pi \mid Y$ is a homeomorphism onto a dense $G_{\delta}$-subset of $\prod \mathrm{Z}_{2}$. We may suppose that $Y$ is invariant under the action of $G$ and of the group generated by $\left(h_{n}\right)(n=1,2, \ldots)$. The theorem now follows by applying Lemma 1.5.

COROLlARY 1.9. Let $G_{j}$ be a countable group for $j=1$ and $j=2$. Let $\alpha_{j}$ be a representation of $G_{j}$ in the group of all homeomorphisms of $S$. Let $\alpha_{j}\left[G_{j}\right]$ act ergodically on $S$.

Then $\alpha_{1}\left[G_{1}\right]$ is equivalent to $\alpha_{2}\left[G_{2}\right]$. There exists a dense $G_{\delta}$-subset of $S, S_{0}$, and there exists a homeomorphism of $S, \phi$, such that

$$
\left\{\left(s, \alpha_{1}^{g}(s)\right): g \in G_{1} \text { and } s \in S_{0}\right\}=\left\{\left(s, \phi \alpha_{2}^{h} \phi^{-1}(s)\right): h \in G_{2} \text { and } s \in S_{0}\right\} .
$$

ProOF. Let $X$ be any perfect Polish space. Then $B(X) / M(X) \approx C(S)$. As remarked in $\S 0$, the results of Maharam and Stone [10] show that each homeomorphism of $S$ is induced by a pseudo-homeomorphism of $X$. For each pseudohomeomorphism $\theta$ of $X$, let $\bar{\theta}$ be the unique homeomorphism of $S$ which is induced by $\theta$. Then, by [10] or see [14, Corollary 2.6], we can find a homomorphism $\beta_{j}$ of $G_{j}$ into the group of pseudo-homeomorphisms of $X$ such that $\overline{\beta_{j}(g)}=\alpha_{j}^{g}$ for each $g \in G_{j}$.

By Theorem $1.8, \beta_{1}\left[G_{1}\right]$ is equivalent to $\beta_{2}\left[G_{2}\right]$. So there exists a pseudohomeomorphism of $X, \gamma$, such that, for every $g$ in $G_{1}, \beta_{1}(g)$ is decomposable with respect to $\left\{\gamma \beta_{2}(h) \gamma^{-1}: h \in G_{2}\right\}$.

Let $\phi=\bar{\gamma}$ so that $\phi$ is a homeomorphism of $S$. Then $\alpha_{1}^{g}$ is decomposable with respect to $\left\{\phi \alpha_{2}^{h} \phi^{-1}: h \in G_{2}\right\}$. Similarly, for each $h$ in $G_{2}, \phi \alpha_{2}^{h} \phi^{-1}$ is decomposable with respect to $\left\{\alpha_{1}^{g}: g \in G_{1}\right\}$. 
2. The $C^{*}$-algebra of an equivalence relation. Throughout this section $X$ is a Hausdorff topological space for which the Baire Category Theorem is true (e.g., a $G_{\delta}$-subset of a compact Hausdorff space, or a complete metric space). Let $\sim$ be an equivalence relation on $X$ such that there exists a countable group $\Gamma$ of homeomorphisms of $X$ such that $x \sim y \Leftrightarrow \gamma \cdot x=y$ for some $\gamma \in \Gamma$.

We shall first construct an algebra in terms of the equivalence relation $\sim$, without explicit mention of $\Gamma$, but we shall make use of the fact that $\sim$ is generated by a countable family of homeomorphisms. In view of the remarks following Proposition 0.1 , this is a mild restriction.

For each $x \in X$, let $[x]$ be the equivalence class generated by $x$; let $[X]$ be the set of all equivalence classes, and let $\mathrm{Gr}$ be the graph of the relation ; let $l^{2}[x]$ be the Hilbert space of all square-summable complex valued functions (sequences) from $[x]$ to $\mathbf{C}$. For each $y \in[x]$, let $\delta_{y}$ be the element of $l^{2}[x]$ such that

$$
\delta_{y}(t)= \begin{cases}1 & \text { for } t=y \\ 0 & \text { for } t \neq y\end{cases}
$$

Then $\left\{\delta_{y}: y \in[x]\right\}$ forms an orthonormal basis for $l^{2}[x]$ which we shall call the canonical orthonormal basis for $l^{2}[x]$.

Let

$$
S=\bigoplus_{[x] \in[X]} \mathcal{L}\left(l^{2}[x]\right)
$$

Here $\mathcal{L}\left(l^{2}[x]\right)$ is the algebra of all bounded operators on $l^{2}[x]$. Clearly $S$ is a Type I von Neumann algebra (being the direct sum of such algebras) and is a subalgebra of the algebra of all bounded operators on the Hilbert space $\bigoplus_{[x] \in[X]} l^{2}[x]$.

The algebra $S$ is not, in itself, of interest, but we shall identify a subalgebra of $S$ with an algebra of "Borel matrices" over $\mathrm{Gr}$ and then form a quotient of the latter algebra.

The set $\mathrm{Gr}$ is the union of the pairwise disjoint sets $[x] \times[x]$; i.e.,

$$
\mathrm{Gr}=\bigcup\{[x] \times[x]:[x] \in[X]\} .
$$

To each operator $M \in S$ we can associate, canonically, a function $m: \mathrm{Gr} \rightarrow \mathbf{C}$ as follows. We have

$$
M=\bigoplus_{[x] \in[X]} M_{[x]}
$$

where $M_{[x]}$ is a bounded operator on $l^{2}[x]$. So each $M_{[x]}$ has a unique matrix representation $m n_{[x]}$ with respect to the canonical orthonormal basis $\left\{\delta_{y}: y \in[x]\right\}$ of $l^{2}[x]$. Thus

$$
m_{[x]}(y, z)=\left\langle M_{[x]} \delta_{z}, \delta_{y}\right\rangle
$$

for all $y, z \in[x]$. Let $m: \mathrm{Gr} \rightarrow \mathbf{C}$ be the function such that

$$
m(x, y)=m_{[x]}(x, y) \quad \text { for all }(x, y) \in \mathrm{Gr} \text {. }
$$

It follows that there is a bijection between elements of $S$ and those functions $m: \mathrm{Gr} \rightarrow \mathbf{C}$ for which there exists a constant $k$ such that, for each $[x] \in[X]$, the restriction of $m$ to $[x] \times[x]$ is the matrix of a bounded operator on $l^{2}[x]$ of norm less than $k$. For such an $m$ let $L(m)$ denote the corresponding element of $S$. 
Manipulation of matrices shows that

$$
L(f) L(g)=L(f \circ g)
$$

where

$$
f \circ g(x, z)=\sum_{y \in[x]} f(x, y) g(y, z)
$$

and

$$
L(f)^{*}=L\left(f^{*}\right)
$$

where $f^{*}(x, y)=\overline{f(y, x)}$, for all $(x, y) \in \mathrm{Gr}$.

We see that, when $f$ is a complex valued function on $\mathrm{Gr}, L(f)$ is a unitary in $S$ if, and only if,

that is, if, and only if,

$$
f^{*} \circ f(x, y)=f \circ f^{*}(x, y)= \begin{cases}1 & \text { for } x=y \\ 0 & \text { for } x \neq y\end{cases}
$$

$$
\sum_{z \in[x]} \overline{f(z, x)} f(z, y)=\sum_{z \in[x]} f(x, z) \overline{f(y, z)}= \begin{cases}1 & \text { for } x=y \\ 0 & \text { for } x \neq y\end{cases}
$$

Since every element of a (unital) $C^{*}$-algebra is a finite linear combination of unitaries, we see that a function $f: \operatorname{Gr} \rightarrow \mathbf{C}$ corresponds to an element of $S$, if, and only if, $f$ is a finite linear combination of "unitary" functions. It follows by a slight refinement of the Russo-Dye Theorem that such an $f$ corresponds to an element of the open unit ball of $S$ if, and only if, $f$ can be expressed in the form $\sum_{1}^{n} \lambda_{j} u_{j}$, where each $u_{j}$ is a "unitary" function and each $\lambda_{j} \geq 0$ and $\sum_{1}^{n} \lambda_{j}<1$.

Let $\left(\gamma_{n}\right)(n=1,2, \ldots)$ be an enumeration of $\Gamma$. For each $n,(x, y) \rightarrow\left(x, \gamma_{n} y\right)$ is a homeomorphism of $X \times X$ onto $X \times X$. Since $X$ is Hausdorff, the diagonal $\Delta=\{(x, x): x \in X\}$ is a closed subset of $X \times X$. Hence $\mathrm{Gr}$ is the union of countably many closed subsets of $X \times X$ and so is a Borel subset of $X \times X$.

Let $M(\mathrm{Gr})$ be the space of all Borel functions $f: \mathrm{Gr} \rightarrow \mathbf{C}$ such that $L(f) \in S$.

LEMMA 2.1. $\{L(f): f \in M(\mathrm{Gr})\}$ is a $C^{*}$-subalgebra of $S$ which is sequentially closed with respect to the weak operator-topology of $S$.

Proof. Let $f, g$ be elements of $M(\mathrm{Gr})$. Since $(x, y) \rightarrow(y, x)$ is a homeomorphism of $X \times X$ onto $X \times X$ we see that, since $f \in M(\mathrm{Gr}), f^{*}$ is also a Borel function on $\mathrm{Gr}$ and hence $f^{*} \in M(\mathrm{Gr})$.

For each $(x, z) \in \mathrm{Gr}$,

$$
f \circ g(x, z)=\sum_{y \in[x]} f(x, y) g(y, z) .
$$

Let $\Delta_{n}=\left\{\left(x, y_{n} x\right): x \in X\right\}$. Because $\Gamma$ is not assumed to act freely, we cannot assume that the sets $\left(\Delta_{n}\right)(n=1,2, \ldots)$ are pairwise disjoint. So we define $D_{n+1}=$ $\Delta_{n+1} \backslash \Delta_{n}$, for $n \geq 1$, and $D_{1}=\Delta_{1}$. Let $f_{n}$ be the Borel function on Gr defined by

$$
f_{n}=\chi_{D_{n}} f
$$

so that $f_{n}$ coincides with $f$ on $D_{n}$ and vanishes outside this set. Then

$$
f \circ g(x, z)=\sum_{n=1}^{\infty} f_{n}\left(x, y_{n} x\right) g\left(y_{n} x, z\right) \text {. }
$$


In order to show that $f \circ g$ is a Borel function on Gr, it suffices to show that, for each $n,(x, z) \rightarrow f_{n}\left(x, y_{n} x\right)$ and $(x, z) \rightarrow g\left(y_{n} x, z\right)$ are Borel functions. This follows from the observation that $(x, z) \rightarrow\left(x, y_{n} x\right)$ is continuous, being the composition of the continuous maps $(x, z) \rightarrow x$ and $x \rightarrow\left(x, y_{n} x\right)$, and that $(x, z) \rightarrow\left(y_{n} x, z\right)$ is also continuous.

Let $\left(b_{n}\right)(n=1,2, \ldots)$ be a sequence in $M(\mathrm{Gr})$ such that $L\left(b_{n}\right) \rightarrow L(b)$ in the weak operator-topology of $S$. Then, for each $(x, y) \in \mathrm{Gr}$,

$$
\left\langle L\left(b_{n}\right) \delta_{x}, \delta_{y}\right\rangle \rightarrow\left\langle L(b) \delta_{x}, \delta_{y}\right\rangle
$$

Thus $b_{n}(y, x) \rightarrow b(y, x)$. So $b$ is a Borel function and hence in $M(\mathrm{Gr})$. This completes the proof of the lemma.

For each $f \in M(\mathrm{Gr})$ let $E f$ be the function on $\mathrm{Gr}$ which vanishes off the diagonal $\Delta$, and such that, for all $x \in X$,

$$
(E f)(x, x)=f(x, x) \text {. }
$$

Clearly $E$ is an idempotent map from $M(\mathrm{Gr})$ onto an abelian subalgebra which we may, and shall, identify with $B(X)$. Let $J$ be the set of all $f$ in $M(\mathrm{Gr})$ for which $E\left(f \circ f^{*}\right)$ vanishes off a meagre subset of $X$. Then it can be proved, by adapting the methods of Feldman and Moore [4], that $J$ is a two-sided ideal of $M(\mathrm{Gr})$ which is sequentially closed in the strong operator-topology of $S$. The quotient algebra $M(\mathrm{Gr}) / J$ we shall call the monotone $C^{*}$-algebra of $(X, \sim)$. However Lemma 2.1 will suffice for the applications in the next section. It will follow immediately from Lemma 3.3 that $J$ is a $\sigma$-ideal of $M(\mathrm{Gr})$, where $\mathrm{Gr}$ is the graph of a countable group of homeomorphisms of $S$.

3. The canonical monotone cross-product $C^{*}$-algebra. As before, $C(S)$ shall be the Dixmier algebra $B(\mathbf{R}) / M(\mathbf{R})$. When $G$ is a countable group of *automorphisms of $C(S)$ which acts freely and ergodically, there is a corresponding monotone cross-product $C^{*}$-algebra, $M(C(S), G)$. This algebra is a Type III $A W^{*}$ factor which contains the Dixmier algebra as a maximal abelian subalgebra and hence is not a von Neumann algebra [11]. Our goal is to show that this algebra is unique. Every free, ergodic action of a countable group on $C(S)$ gives rise to the same $A W^{*}$-factor.

We shall begin with some remarks on the Hamana tensor product. More detailed information can be found in $[\mathbf{6}, \mathbf{7}, \mathbf{1 2}]$.

From now on, $H$ is a separable Hilbert space and $H_{1}$ an arbitrary Hilbert space. Let us fix an orthonormal basis for $H$. Then, with respect to this basis, every $x$ in $\mathcal{L}\left(H_{1}\right) \bar{\otimes} \mathcal{L}(H)$ has a unique representation as a matrix $\left[x_{i j}\right]$, where each $x_{i j}$ is in $\mathcal{L}\left(H_{1}\right)$. Let $M$ be a von Neumann subalgebra of $\mathcal{L}\left(H_{1}\right)$. Then the elements of $M \bar{\otimes} \mathcal{L}(H)$ are those elements of $\mathcal{L}\left(H_{1}\right) \bar{\otimes} \mathcal{L}(H)$ represented by matrices $\left[m_{i j}\right]$ where each $m_{i j}$ is in $M$.

Let $T$ be any set and $\operatorname{Bnd}(T)$ the commutative von Neumann algebra of all bounded complex functions on $T$. Let $T$ be given the discrete topology, and let $\beta T$ be its Stone-Cech compactification. Then $\beta T$ is extremally disconnected and $\operatorname{Bnd}(T) \approx C(\beta T)$. By Lemma $1.1[\mathbf{7}] \operatorname{Bnd}(T) \bar{\otimes} \mathcal{L}(H)$ can be identified with $C_{w}(\beta T, \mathcal{L}(H))$, the space of continuous functions from $\beta T$ to $\mathcal{L}(H)$ equipped with the weak operator-topology. Let $F: T \rightarrow \mathcal{L}(H)$ have (norm) bounded range. Then, 
since the unit ball of $\mathcal{L}(H)$ is compact in the weak-operator topology, the StoneCech Theorem [8, p. 153] implies that $F$ has a unique extension to a continuous map $\hat{F}: \beta T \rightarrow \mathcal{L}(H)$. So we may identify $\operatorname{Bnd}(T) \bar{\otimes} \mathcal{L}(H)$ with the algebra of all matrices $\left[m_{i j}\right]$ over $\operatorname{Bnd}(T)$ for which $t \rightarrow\left[m_{i j}(t)\right]$ is a bounded function on $T$.

From now on, $X$ is a topological space which is either Polish or homeomorphic to a $G_{\delta}$-subset of a compact Hausdorff space. We recall that $B(X)$ is the commutative algebra of bounded Borel functions on $X$. The product $B(X) \tilde{\otimes} \mathcal{L}(H)$ may be defined as the Borel *-envelope of $B(X) \otimes_{\min } \mathcal{L}(H)$ inside $\operatorname{Bnd}(X) \bar{\otimes} \mathcal{L}(H)$ (see [11]). The elements of $B(X) \tilde{\otimes} \mathcal{L}(H)$ correspond to the matrices $\left[b_{i j}\right]$ where each $b_{i j}$ is in $B(X)$ and the map $x \rightarrow\left\|\left[b_{i j}(x)\right]\right\|$ is bounded on $X$.

When $G$ is a countable group of homeomorphisms of $X$, then $M^{\sigma}(B(X), G)$ is defined to be the subalgebra of $B(X) \tilde{\otimes}\left(l^{2}(G)\right)$ consisting of those elements of the tensor product which have a matrix representation over $B(X)$ of the form $\left[a_{\gamma, \sigma}\right](\gamma \in G, \sigma \in G)$, where $a_{\gamma \tau, \sigma \tau}(x)=a_{\gamma, \sigma}(\tau x)$ for all $x \in X$ and all $\tau \in G$.

LEMMA 3.1. Assume that each $\gamma \in G$ has no fixed points in $x$ unless $\gamma$ is the identity. Let $\mathrm{Gr}$ be the graph of $G$. Then $M(\mathrm{Gr})$ is naturally *-isomorphic to $M^{\sigma}(B(X), G)$.

Proof. Let $f \in M(\mathrm{Gr})$. For each $\gamma, \sigma$ in $G$ let $a_{\gamma, \sigma}(x)=f(\gamma x, \sigma x)$. Then $a_{\gamma, \sigma}$ is in $B(X)$. Also the norm of $\left[a_{\gamma, \sigma}(x)\right]$ is uniformly bounded for $x$ in $X$. Hence $\left[a_{\gamma, \sigma}\right]$ is in $B(X) \tilde{\otimes} \mathcal{L}\left(l^{2}(G)\right)$. Moreover, for all $\gamma, \sigma, \tau$ in $G$ and all $x \in X$,

$$
a_{\gamma \tau, \sigma \tau}(x)=f(\gamma \tau x, \sigma \tau x)=a_{\gamma, \sigma}(\tau x) .
$$

Thus $\left[a_{\gamma, \sigma}\right]$ is in $M^{\sigma}(B(X), G)$.

Conversely, let $\left[a_{\gamma, \sigma}\right]$ be in $M^{\sigma}(B(X), G)$. Because of our hypothesis on the action of $G$, we may define a function $f: \mathrm{Gr} \rightarrow \mathbf{C}$ by

$$
f(x, \tau x)=a_{e, \tau}(x) \text { for all } \gamma \in G \text { for all } x \in X .
$$

From the definition of $M^{\sigma}(B(X), G)$, for any $\gamma, \sigma$ in $G$ and any $x$ in $X$,

$$
a_{\gamma, \sigma}(x)=a_{e, \sigma \gamma^{-1}}(\gamma x)=f(\gamma x, \sigma x) .
$$

It is now easy to see that $f$ is in $M(\mathrm{Gr})$. Matrix manipulations show that this bijection is a $*$-isomorphism of $M(\mathrm{Gr})$ onto $M^{\sigma}(B(X), G)$.

Let $A$ be any commutative $A W^{*}$-algebra. Let $G$ be a countable group of *automorphisms of $A$. Then $A \approx C(Y)$, where $Y$ is compact and extremally disconnected. We shall abuse our notation and also regard $G$ as a group of homeomorphisms of $Y$.

We recall that a *-automorphism, $h$, of $A$ is said to be properly outer if there does not exist a nonzero projection $e$ in $A$ such that the restriction of $h$ to $e A$ is the identity map. The action of $G$ on $A$ is said to be free if every element of $G$, other than the identity, is a properly outer automorphism.

We shall now suppose that the action of $G$ on $A$ is free.

Let $\gamma$ be any element of $G$ other than the identity. Let $F_{\gamma}$ be the closed set $\{t \in Y: \gamma t=t\}$. Since $Y$ is extremally disconnected, the interior of $F_{\gamma}$ is clopen. It now follows from the freeness of the action of $G$ that the interior of $F_{\gamma}$ is empty; that is, $F_{\gamma}$ is nowhere dense. Since $G$ is countable, the $G$-saturation of $\bigcup\left\{F_{\gamma}: \gamma \in G\right.$ and $\gamma$ is not the identity $\}$ is a meagre $F_{\sigma}$-set. So there is a $G$-invariant dense $G_{\delta}$-subset, 
$Y_{0}$, of $Y$, such that, whenever $g \in G$ and $g$ is not the identity, then $g$ has no fixed points in $Y_{0}$.

Let $\pi$ be the quotient homomorphism from $B\left(Y_{0}\right)$ onto $C(Y)$ whose kernel is $\operatorname{Mg}\left(Y_{0}\right)$. Each element of the Hamana tensor product $C(Y) \bar{\otimes} \mathcal{L}\left(l^{2}(G)\right)$ has a representation as a matrix over $C(Y)$. (Warning: The multiplication in $C(Y) \bar{\otimes} \mathcal{L}\left(l^{2}(G)\right.$ ) is not straightforward.) By Theorem $2.5[12]$ there exists a canonical $\sigma$-normal *-homomorphism $\Pi$ from $B\left(Y_{0}\right) \tilde{\otimes} \mathcal{L}\left(l^{2}(G)\right)$ onto $C(Y) \bar{\otimes} \mathcal{L}\left(l^{2}(G)\right)$ such that

$$
\Pi\left(\left[a_{\gamma \sigma}\right]\right)=\left[\pi\left(a_{\gamma \sigma}\right)\right] \text {. }
$$

The monotone cross-product of $C(Y)$ by $G, M(C(Y), G)$, is the subalgebra of $C(Y) \bar{\otimes} \mathcal{L}\left(l^{2}(G)\right)$ corresponding to those matrices $\left[a_{\sigma, \gamma}\right]$ over $C(Y)$ which satisfy the identities $a_{\sigma \tau, \gamma \tau}(s)=a_{\sigma, \gamma}(\tau s)$ for all $\sigma, \gamma, \tau$ in $G$ and all $s \in Y$.

Saitô [11] gives a very lucid account of monotone cross-products and also of the results of Takenouchi and Dyer. He only discusses cross-products by abelian groups, but everything extends to nonabelian groups without difficulty.

LEMMA 3.2. The homomorphism $\Pi$ maps $M^{\sigma}\left(B\left(Y_{0}\right), G\right)$ onto $M(C(Y), G)$.

This is straightforward.

The diagonal algebra of a monotone cross-product $M(C(Y), G)$ consists of those elements whose matrix is of the form $\left[a_{\gamma, \sigma}\right]$, where $a_{\gamma, \sigma}=0$ for $\gamma \neq \sigma$. This is clearly isomorphic to $C(Y)$. Let $\mathrm{Gr}$ be the graph of the orbit equivalence relation on $Y_{0}$ given by the action of $G$. Then the diagonal algebra of $M(\mathrm{Gr})$ consists of those $f$ in $M(\mathrm{Gr})$ which vanish off the diagonal of $\mathrm{Gr}$. The operator $E$ maps $M(\mathrm{Gr})$ onto its diagonal algebra. Clearly the diagonal algebra of $M(\mathrm{Gr})$ may be identified with $B\left(Y_{0}\right)$.

LEMMA 3.3. Let $\mathrm{Gr}$ be the graph of the relation of orbit equivalence given by $G$ acting on $Y_{0}$. Then there exists a $\sigma$-normal *-homomorphism $\rho$ from $M(\mathrm{Gr})$ onto $M(C(Y), G)$. The kernel of $\rho$ is

$$
J=\left\{z \in M(\mathrm{Gr}): E\left(z z^{*}\right) \text { vanishes off a meagre subset of } Y_{0}\right\} .
$$

Furthermore, $\rho$ maps the diagonal subalgebra of $M(\mathrm{Gr})$ onto the diagonal algebra of $M(C(Y), G)$.

PROOF. The existence and $\sigma$-normality of $\rho$ follows immediately from Lemmas 3.1 and 3.2. Let $J_{0}$ be the kernel of $\rho$.

Then $f$ will be in $J_{0}$ if, and only if, the function $x \rightarrow \sum_{\tau \in G}|f(x, \tau x)|^{2}$ vanishes off a meagre subset of $Y_{0}$. That is, $f$ is in $J$ if, and only if, $E\left(f \circ f^{*}\right)$ vanishes off a meagre subset of $Y_{0}$. Thus $J_{0}=J$, as required.

We come now to the main theorem.

THEOREM 3.4. Let $D$ be the Dixmier algebra. Let $G_{1}$ and $G_{2}$ be countable groups of *-automorphisms of $D$ which both act freely and ergodically. Then there exists an isomorphism of $M\left(D, G_{1}\right)$ onto $M\left(D, G_{2}\right)$ which maps the diagonal algebra of $M\left(D, G_{1}\right)$ onto the diagonal algebra of $M\left(D, G_{2}\right)$.

We may regard $G_{1}$ and $G_{2}$ as groups of homeomorphisms of $S$. Because the actions are free, there exists a dense $G_{\delta}$-subset $S_{0}$, which is invariant under $G_{1}$ and $G_{2}$, such that only the identity elements of $G_{1}$ and $G_{2}$ have a fixed point in $S_{0}$. 
Since $G_{1}$ and $G_{2}$ act ergodically, it follows from Corollary 1.9 that we can suppose that there exists a *-automorphism $\theta$ of $D$ such that the graph of $G_{1}$ acting on $S_{0}$ coincides with the graph of $\theta G_{2} \theta^{-1}$ acting on $S_{0}$. Let $\mathrm{Gr}$ be this graph. Since $M\left(D, G_{2}\right)$ is naturally isomorphic to $M\left(D, \theta G_{2} \theta^{-1}\right)$ we shall suppose that $\theta$ is the identity. (For $\theta \overline{\otimes i d}$ is a $*$-automorphism of $D \bar{\otimes} \mathcal{L}\left(\rho^{2}(G)\right)$ which induces the required natural isomorphism.) Then, by Lemma 3.3, there exists an isomorphism $\rho_{j}$ from $M(\mathrm{Gr}) / J$ onto $M\left(C(S), G_{j}\right)$ for $j=1,2$. Then $\rho_{2} \rho_{1}^{-1}$ is the required isomorphism from $M\left(D, G_{1}\right)$ onto $M\left(D, G_{2}\right)$.

COROLLARY 3.5. The Takenouchi factor is isomorphic to the Dyer factor.

PROOF. The Takenouchi factor is of the form $M\left(D, G_{1}\right)$, where $G_{1}$ is generated by an irrational rotation of the circle. The Dyer factor is $M\left(D, G_{2}\right)$, where $G_{2}$ is the group of dyadic rationals acting on $\mathbf{R}$ modulo 1 .

\section{REFERENCES}

1. A. Connes, J. Feldman and B. Weiss, Amenable equivalence relations generated by a single transformation, Ergodic Theory Dynamical Systems 1 (1981), 431-450.

2. J. Dyer, Concerning $A W^{*}$-algebras, Notices Amer. Math. Soc. 17 (1970), 788; Abstract \#677-47-5.

3. J. Feldman and Calvin C. Moore, Ergodic equivalence relations, cohomology, and von Neumann algebras. I, Trans. Amer. Math. Soc. 234 (1977), 289-324.

4. __ Ergodic equivalence relations, cohomology, and von Neumann algebras. II, Trans. Amer. Math. Soc. 234 (1977), 325-359.

5. J. Feldman, Amenability, hyperfiniteness, and Cartan sub-algebras, Operator Algebras and Group Representations (Proc. Internat. Conf., Romania, 1980), Vol. 1, Pitman, London, 1984, pp. 185-191.

6. M. Hamana, Tensor products for monotone complete $C^{*}$-algebras. I, Japan J. Math. 8 (1982), 261-283.

7. _ Tensor products for monotone complete $C^{*}$-algebras. II, Japan J. Math. 8 (1982), 285-295.

8. J. Kelley, General topology, Van Nostrand, Princeton, N. J., 1955.

9. K. Kuratowski, Topology, Vol. 1, Academic Press, New York, 1966.

10. D. Maharam and A. H. Stone, Realising isomorphisms of category algebras, Bull. Austral. Math. Soc. 19 (1978), 5-10.

11. K. Saitô, $A W^{*}$-algebras with monotone convergence properties and examples by Takenouchi and Dyer, Tôhoku Math. J. 31 (1979), 31-40.

12. K. Saitô and J. D. M. Wright, On tensor products of monotone complete algebras, Quart. J. Math. Oxford Ser. (2) 35 (1984), 209-221.

13. R. Sikorski, Boolean algebras, 2nd ed., Springer-Verlag, Berlin, 1964.

14. D. Sullivan and J. D. M. Wright, On lifting automorphisms of monotone $\sigma$-complete $C^{*}$ algebras, Quart. J. Math. Oxford Ser. (2) 32 (1981), 371-381.

15. O. Takenouchi, $A$ non- $W^{*}, A W^{*}$-factor, $C^{*}$-Algebras and Applications to Physics (Proc. Second Japan-U.S.A. Sem., Los Angeles, 1977), Lecture Notes in Math., vol 650, SpringerVerlag, Berlin and New York, 1978, pp. 135-139.

16. J. D. M. Wright, Wild AW*-factors and Kaplansky-Rickart algebras, J. London Math. Soc. 13 (1976), 83-89.

Department of MAThematics, City ŨNIVERSity of NeW YoRk, NeW York NeW YORK 10036

INSTITUTE OF MATHEMATICS AND COMPUTER SCIENCE, HEBREW UNIVERSITY OF JERUSALEM, GIVAT RAM, 91904 JERUSALEM, ISRAEL

DEPARTMENT OF MATHEMATICS, UNIVERSiTY OF READING, WHITEKNIGHTS, PO BOX 220, READING, ENGLAND RG6 2AX 